\title{
Dentin deproteinization effect on bond strength of self-adhesive resin cements
}

\section{Danielle Sampaio Lisboa ${ }^{(a)}$ Silvando Vieira dos Santos ${ }^{(\mathrm{b})}$ Sandro Griza ${ }^{(b)}$ João Lima Rodrigues(c) André Luis Faria-e-Silva(a)}

(a) Department of Dentistry, School of Dentistry, Univ Federal de Sergipe - UFS, Aracaju, SE, Brazil.

(b) Department of Materials Science and Engineering, Univ Federal de Sergipe - UFS, São Cristóvão, SE, Brazil.

(c) Department of Dentistry, Faculdades Integradas do Norte de Minas - FUNORTE, Montes Claros, MG, Brazil.

Declaration of Interests: The authors certify that they have no commercial or associative interest that represents a conflict of interest in connection with the manuscript.

Corresponding Author:

André Luis Faria-e-Silva

E-mail: fariaesilva.andre@gmail.com

Submitted: Jul 30, 2012

Accepted for publication: Oct 01, 2012

Last revision: Oct 09, 2012
Abstract: This study examined the effect of deproteinization on the bond strength between self-adhesive resin cements and dentin surfaces that were untreated (control), acid-etched, or acid-etched and subjected to a post-etch deproteinization treatment. Cylinders of RelyX Unicem or BisCem $(n=6)$ cement were build-up on the dentin surfaces and tested to determine shear strength. The results were analyzed using two-way ANOVA and Tukey's test (5\%). While neither dentin pretreatment improved the bond strength for RelyX Unicem, deproteinization treatments resulted in greater bond strength in BisCem specimens while acid etching alone did not improve the performance of the material.

Descriptors: Dentin; Resin Cements; Shear Strength; Sodium Hypochlorite.

\section{Introduction}

Self-adhesive resin cements (SARCs) were developed to simplify clinical procedures and overcome the technique sensitivity associated with the use of multi-step systems. According to their manufacturers, SARCs do not require pretreatment of tooth surfaces and their application is completed in a single clinical step. Despite this attractive concept, limited etching potential and superficial interaction with dentin have been observed in some self-adhesive resin cements. ${ }^{1,2}$ In addition, the mineral components of the smear layer are effective buffers, ${ }^{3}$ making the $\mathrm{pH}$ at the interface too high to demineralize the underlying dentin. ${ }^{4}$ Workers investigating pretreatment of dentin with acid solutions have reported that removing the smear layer improved the interaction between SARCs and dentin. ${ }^{4-7}$ However, the results of these studies were often material dependent, and pretreatment with phosphoric acid resulted in reduction of the bond strength since the presence of collagen fibrils reduced the penetration of the cement. ${ }^{7}$ It is therefore expected that dentin deproteinization with collagen removal will increase the contact between SARCs and dentin. We evaluated the effect of dentin deproteinization on the SARC/ dentin bond strength under the null hypothesis that dentin deproteinization does not affect the strength of the bond.

\section{Methodology}

The specimens consisted of 36 extracted sound human third molars. The occlusal surfaces were ground flat using 180-, 320-, and 600-grit silicon carbide paper under running water to remove the enamel and ex- 
pose a flat dentin surface. The dentin surface was treated according to one of the following protocols:

- Control - no treatment;

- Acid etching - etching with 34\% phosphoric acid (3M ESPE, St. Paul, USA) for $15 \mathrm{~s}$, followed by rinsing with water for $10 \mathrm{~s}$ and drying with $\mathrm{ab}-$ sorbent paper;

- Deproteinization - after acid etching as previously described, deproteinization with $5 \% \mathrm{NaOCl}$ for 2 minutes, rinsing with water for $10 \mathrm{~s}$, and drying under a stream of compressed air for $10 \mathrm{~s}$.

Polyvinylsiloxane molds (Aquasil Extra Low Viscosity, Dentsply DeTrey, Konstanz, Germany) containing three cylindrical cavities $1 \mathrm{~mm}$ in diameter and $2 \mathrm{~mm}$ tall were placed on the dentin surface. The mold cavities were filled with RelyX Unicem (3M ESPE, St. Paul, USA) or BisCem (Bisco, Schaumburg, USA) cement. The specimens were lightcured for $20 \mathrm{~s}$ using a light-emitting diode unit (Radii Cal, SDI, Bayswater, Australia) with an irradiance of $800 \mathrm{~mW} / \mathrm{cm}^{2}$. After $24 \mathrm{~h}$, shear bond tests were conducted using a universal testing machine (Instron 5565, Instron, Canton, USA). A thin steel wire $(0.2 \mathrm{~mm}$ diameter) was looped around each cylinder and a shear load was applied to the base of the cylinder at a crosshead speed of $0.5 \mathrm{~mm} /$ min until failure. The average load at failure of the three cylinders was recorded as the microshear bond strength (MPa) for that specimen. The shear test results were subjected to two-way ANOVA and the post-hoc Tukey's test $(\alpha=0.05)$. Following the shear tests, the dentin surfaces were examined using an optical microscope at $40 \times$ magnification.

\section{Results}

The bond strength test results are presented in Table 1. The factor "dentin treatment" was not significant $(\mathrm{p}=0.169)$, whereas the factor "cement" $(\mathrm{p}<0.001)$ and the interaction between the two factors $(\mathrm{p}=0.017$ ) were both significant. For RelyX Unicem samples, there was no difference between the dentin treatments. For BisCem samples, dentin deproteinization resulted in significantly higher bond strength than the control group $(\mathrm{p}=0.005)$. Acid etching did not produce a significant difference
Table 1 - Mean (standard deviation) shear bond strengths in MPa for resin-dentin samples.

\begin{tabular}{c|c|c}
\hline \multirow{2}{*}{ Dentin treatment } & \multicolumn{2}{|c}{ Self-adhesive resin cement } \\
\cline { 2 - 3 } & Unicem & BisCem \\
\hline None (control) & $13.7(4.8)^{A, a}$ & $3.6(1.1)^{\mathrm{B}, \mathrm{b}}$ \\
\hline Acid etching & $14.2(2.7)^{\mathrm{A}, \mathrm{a}}$ & $5.3(1.3)^{\mathrm{B}, \mathrm{ab}}$ \\
\hline Deproteinization & $12.6(3.5)^{\mathrm{A}, \mathrm{a}}$ & $9.7(3.7)^{\mathrm{A}, \mathrm{a}}$ \\
\hline
\end{tabular}

Distinct uppercase letters in the same line indicate differences between cements; distinct lowercase letters in the same column indicate differences between treatments $(P<0.05)$.

from the control $(\mathrm{p}=0.611)$ or deproteinization $(\mathrm{P}=0.053)$ treatments. The mean values obtained for the acid-etched group were statistically similar to those obtained for both the control and deproteinized groups. Except for the deproteinized samples, RelyX Unicem exhibited greater bond strength than BisCem. Adhesive failure was the primary failure mechanism under all conditions.

\section{Discussion}

When compared to the control group results, dentin deproteinization did not improve the performance of RelyX U-100 but did improve the bond strength of BisCem, resulting in rejection of the null hypothesis. Several studies have demonstrated the limited ability of SARCs to demineralize and dissolve the smear layer to reach the underlying dentin..$^{1-7}$ This limitation is attributed to the high viscosity $^{8}$ of the cements and the buffering effect that takes place during their setting reaction. ${ }^{9}$ The smear layer on the dentin substrate of the present study was produced using 600-grit silicon carbide paper, and it has previously been demonstrated that smear layers produced using this method are thicker than layers obtained using a carbide bur (approximately $1.8 \mu \mathrm{m}) .{ }^{10}$

In a previous study, pretreatment with phosphoric acid reduced the bond strength of RelyX Unicem to dentin. ${ }^{7}$ Despite smear layer removal, the thick collagen mesh exposed by acid etching prevented the viscous cement from reaching the deeper unaffected dentin. ${ }^{7}$ In contrast, preliminary acid etching did not alter the bond strength of RelyX Unicem to dentin in the present study. Similar behavior was also observed in the BisCem specimens. One difference 
between the studies was that in the present study, excess water was removed using absorbent paper rather than an air stream. Retention of a greater amount of water possibly resulted in larger spaces between the collagen fibrils, ${ }^{11}$ improving penetration of the cement.

A combination of acid etching followed by dentin deproteinization and exposed collagen removal using $\mathrm{NaOCl}$ improved the bond strength of BisCem to dentin, but did not improve the performance of RelyX Unicem. In addition to smear layer removal, collagen removal theoretically promotes more

\section{References}

1. Al-Assaf K, Chakmakchi M, Palaghias G, Karanika-Kouma A, Eliades G. Interfacial characteristics of adhesive luting resins and composites with dentine. Dent Mater. 2007 Jul;23(7):82939.

2. Monticelli F, Osorio R, Mazzitelli C, Ferrari M, Toledano M. Limited decalcification/diffusion of self-adhesive cements into dentin. J Dent Res. 2008 Oct;87(10):974-9.

3. Kenshima S, Reis A, Uceda-Gomez N, Tancredo LL, Filho LE, Nogueira FN, et al. Effect of smear layer thickness and $\mathrm{pH}$ of self-etching adhesive systems on the bond strength and gap formation to dentin. J Adhes Dent. 2005 Summer;7(2):117-26.

4. Mazzitelli C, Monticelli F, Toledano M, Ferrari M, Osorio R. Dentin treatment effects on the bonding performance of selfadhesive resin cements. Eur J Oral Sci. 2010 Feb;118(1):80-6.

5. Pavan S, Santos PH, Berger S, Bedran-Russo AK. The effect of dentin pretreatment on the microtensile bond strength of selfadhesive resin cements. J Prosthet Dent. 2010 Oct;104(4):25864.

6. Santos MJ, Bapoo H, Rizkalla AS, Santos GC. Effect of dentin-cleaning techniques on the shear bond strength of intimate contact between the SARCs and dentin. However, dentin deproteinization also creates a hydrophilic surface that could hinder the interaction of more hydrophobic materials such as the BisGMA monomer present in Unicem, reducing the overall wetting ability of the cement.

\section{Conclusion}

Deproteinization improved the bond strength of BisCem to dentin but did not improve the performance of RelyX Unicem when compared to untreated dentin specimens.

self-adhesive resin luting cement to dentin. Oper Dent. 2011 Sep-Oct;36(5):512-20.

7. De Munck J, Vargas M, Van Landuyt K, Hikita K, Lambrechts $\mathrm{P}$, Van Meerbeek B. Bonding of an auto-adhesive luting material to enamel and dentin. Dent Mater. 2004 Dec;20(10):96371.

8. Burke FJ, Crisp RJ, Richter B. A practice-based evaluation of the handling of a new self-adhesive universal resin luting material. Int Dent J. 2006 Jun;56(3):142-6.

9. Han L, Okamoto A, Fukushima M, Okiji T. Evaluation of physical properties and surface degradation of self-adhesive resin cements. Dent Mater J. 2007 Nov;26(6):906-14.

10. Oliveira SS, Pugach MK, Hilton JF, Watanabe LG, Marshall SJ, Marshall GW Jr. The influence of the dentin smear layer on adhesion: a self-etching primer vs. a total-etch system. Dent Mater. 2003 Dec;19(8):758-67.

11. Bertassoni LE, Orgel JP, Antipova O, Swain MV. The dentin organic matrix - limitations of restorative dentistry hidden on the nanometer scale. Acta Biomater. 2012 Jul;8(7):2419-33. 